\title{
Mining for Observables: A New Challenge in Numerical Relativity
}

\author{
Deirdre Shoemaker*, Harald Pfeiffer ${ }^{\dagger}$, Larry Kidder**, Pablo Laguna ${ }^{\ddagger}$, Lee \\ Lindblom $^{\dagger}$, Mark Scheel ${ }^{\dagger}$ and Saul Teukolsky** \\ * Department of Physics, Institute for Gravitational Physics and Geometry, Penn State, University \\ Park PA 16802 \\ ${ }^{\dagger}$ Theoretical Astrophysics 130-33, California Institute of Technology, Pasadena CA 91125 \\ ${ }^{* *}$ Center for Radiophysics and Space Research, Cornell University, Ithaca NY 14853 \\ ${ }^{\ddagger}$ Department of Astronomy and Astrophysics, Institute for Gravitational Physics and Geometry, \\ Penn State, University Park PA 16802
}

\begin{abstract}
One of the motivations behind numerical relativity is to provide gravitational wave signals of compact objects to observers using the new gravitational wave detectors. Yet, because of the complexities involved, no dependable signals of binary-black hole coalescences have been established. The work in this proceedings is motivated by how numerical relativity can be used today to predict robust features in gravitational wave signals of binary black-hole coalescence by making approximations to the full problem. To illustrate this, we present results from evolving a Klein-Gordon equation on a frozen background. The background is set by a sequence of initial data in which the binary is in quasi-equilibrium. We probe the data resulting from the evolution for the transition between the linear and non-linear regimes using oscillations of the black holes as our guide. This information is used to motivate a qualitative picture of the gravitational signal of a black-hole coalescence.
\end{abstract}

Keywords: numerical relativity, black holes, gravitational waves

PACS: 04.25.Dm, 04.30.Db, 04.70.Bw, 95.30.Sf, 97.60.Lf

\section{INTRODUCTION}

Binary black holes are excellent candidates of detection by ground and space-based gravitational-wave observatories. The success of ground-based detectors may depend on the quality of modeled waveforms due to the low signal-to-noise ratios. Numerical relativity's goals are to model general relativistic sources that are strongly non-linear, of which the binary black hole coalescence is an excellent example. These models will provide information for gravitational wave detection and characterization. In the absence of an exhaustive set of waveforms, numerical relativity needs to provide information abbout the robust features of binary black hole coalescence to aid in the detection of the gravitational waves. The binary black hole coalescence problem is traditionally broken down into three epochs including inspiral, merger and ringdown. The inspiral phase is a slow adiabatic inspiral of the two black holes, and has been successfully modeled with post-Newtonian methods [1]. The merger phase involves the highly non-linear dynamics of curved spacetime and necessitates a heavily computational approach. The ringdown phase is dominated by an exponentially damped sinusoid and includes information about the mass and spin of the final black hole.

Recently, the numerical relativity community has made great strides in producing

CP848, Recent Advances in Astronomy and Astrophysics,

$7^{\text {th }}$ International Conference of the Hellenic Astronomical Society, edited by N. H. Solomos

(C) 2006 American Institute of Physics 0-7354-0343-0/06/\$23.00 
reliable simulations of the binary black hole coalescence $[2,3,4,5,6,7,8,9]$. The startling success of the past few months has seen the first orbits of binary black holes $(\mathrm{BBH})$, orbits through to merger and the generation of a series of waveforms involving equal mass and unequal mass [8] black holes. In the next few years, we will see the range of parameter space studied increase to include spinning black holes, more astrophysically relevant mass ratios and increasing black-hole separations. Although not-exhaustive, already a picture of the key features of the binary black hole coalescence is emerging, a picture of the $\mathrm{BBH}$ gravitational wave that is strikingly simple.

There have been hints at the simplicity of the waveforms produced by an equal-mass binary black hole orbit and collision from both the close-limit [10, 11] and Lazarus [12] approaches. One of the remarkable outcomes of the close-limit approach to BBH collisions was the range of agreement that these results had with non-linear numerical calculations [13]. Close-limit results closely followed the non-linear simulations beyond the point where the close-limit approximation was in principle valid. The reason for this agreement is not well understood. One possible explanation, which is the main motivation of this work, is that perhaps a potential barrier emerges hiding the non-linear dynamics taking place. The Lazarus approach took the close-limit work further by combining perturbative calculations with the then short-lived, fully non-linear computations to produce the first waveforms from plunge-like BBH dynamics. As the ability to evolve the final orbit and plunge has emerged, the Lazarus work has been surprisingly correct in its prediction of the bulk features of the gravitational waves from a binary black hole coalescence.

A comparison between the results of close-limit, Lazarus and full numerical relativity suggests that one of the key features leaving an imprint on the emitted gravitational radiation is the ringing of the potential. For a single black hole, a potential is clearly identifiable. The well-known quasi-normal ringing and power-law tail behavior is associated with the presence of this potential [14]. Because quasi-normal modes (QNMs) provide unique complex frequencies depending only on the black-hole mass and spin (we will assume neutral black holes for this paper), the QNMs can act as probes of the black hole spacetime.

One can then speculate that, if a potential barrier is formed before the BBH system reaches ISCO, this barrier could unfortunately hide rich non-linear signatures in the emitted gravitational radiation. The signal would be dominated by the ringing modes of this potential. A starting point to address this issue is to study the dynamics of a scalar field on the "frozen" space-time of a BBH system. The scalar field acts as a tracer to detect and map the potential.

\section{KLEIN-GORDON EQUATION}

We solve the Klein-Gordon equation, $\square \psi=0$, on a curved background using the Cornell/Caltech spectral code [15]. Following Ref. [16], we rewrite this equation in a firstorder symmetric hyperbolic form. This is accomplished by introducing the following variables:

$$
\Pi \equiv-\frac{1}{\alpha}\left(\partial_{t} \psi-\beta^{i} \partial_{i} \psi\right)
$$




$$
\Phi_{i} \equiv \partial_{i} \psi
$$

In terms of these variables, the Klein-Gordon equation $\square \psi=0$ can be written as

$$
\begin{aligned}
\left(\partial_{t}-\beta^{j} \partial_{j}\right) \psi & =-\alpha \Pi \\
\left(\partial_{t}-\beta^{j} \partial_{j}\right) \Pi & =-\alpha g^{i j} \partial_{j} \Phi_{i}+\alpha \Gamma^{i} \Phi_{i} \\
& -g^{i j} \partial_{j} \alpha \Phi_{i}+\alpha \Pi K \\
\left(\partial_{t}-\beta^{j} \partial_{j}\right) \Phi_{i} & =-\alpha \partial_{i} \Pi+\Phi_{j} \partial_{i} \beta^{j}-\Pi \partial_{i} \alpha
\end{aligned}
$$

where

$$
\begin{aligned}
\Gamma^{i} & \equiv g^{j k} \Gamma_{j k}^{i} \\
& =-\partial_{j} g^{i j}-g^{i j} \partial_{j} \ln \sqrt{g}
\end{aligned}
$$

Eqs. (3-5) can be rewritten as

$$
\partial_{t} u+A^{i} \partial_{i} u=F
$$

where

$$
\begin{gathered}
\phi=\left(\begin{array}{c}
\psi \\
\Pi \\
\Phi_{j}
\end{array}\right) \\
A^{i}=\left(\begin{array}{ccc}
-\beta^{i} & 0 & 0 \\
0 & -\beta^{i} & \alpha g^{i j} \\
0 & \alpha \delta_{j}^{i} & -\beta^{i}
\end{array}\right)
\end{gathered}
$$

and

$$
F=\left(\begin{array}{c}
-\alpha \Pi \\
\alpha \Gamma^{i} \Phi_{i}-g^{i j} \partial_{j} \alpha \Phi_{i}+\alpha \Pi K \\
\Phi_{i} \partial_{j} \beta^{i}-\Pi \partial_{j} \alpha
\end{array}\right) .
$$

If $\xi^{i}$ is a unit normal to any two-dimensional surface, the characteristic speeds and fields can be found from the eigenvalues and eigenfields of the matrix $A^{i} \xi_{i}$. The characteristic fields are:

$$
\begin{aligned}
u^{ \pm} & =\Pi \pm \xi^{i} \psi_{i} \\
u_{i} & =\psi_{i}-\xi_{i} \xi^{j} \psi_{j} \\
u^{\psi} & =\psi .
\end{aligned}
$$

The fields $u^{ \pm}$have characteristic speeds $-\beta^{i} \pm \alpha \xi^{i}$ (speed of light). On the other hand, the fields $u_{i}$ and $u^{\psi}$ have characteristic speed $-\beta^{i}$ (zero velocity, normal to the spatial hypersurface). One can see from these equations that the information from the background that must be supplied is $g_{i j}, K, \alpha$ and $\beta^{i}$. The next section discusses our choice for the background quantities. 


\section{BINARY BLACK HOLE SPACETIME AS A CURVED BACKGROUND}

We need to specify the background for the Klein-Gordon equation given by $g_{i j}, K$, $\alpha$ and $\beta^{i}$. To study the formation of a potential during the $\mathrm{BBH}$ coalescence, ideally we would use the geometry given by a numerical calculation of the binary system. In this proceedings, we use an approximation to the spacetime of two black holes in orbit given by the quasi-circular sequence of initial data generated by the thin-sandwich $[17,18,19]$. The thin-sandwich method of initial data provides us with $g_{i j}, K, \alpha$ and $\beta^{i}$ for the background. The coordinates are co-rotating with the black holes such that the black holes remain stationary for timescales $T_{\text {orbit }}<<T_{\text {radiationreaction }}$ Table (1) lists the details about wach initial data set in the sequence of quasi-circular equilibrium. The data is constructed using Harald Pfeiffer's multigrid, spectral code for solving elliptic equations [20].

TABLE 1. Parameters for each initial data set in a quasi-circular sequence. The first column is $d$, separation, the second is the orbital frequency, $\Omega M_{i r r}$, the third is the irreducible mass, $M_{i r r}=\sqrt{\frac{A_{A H}}{16 \pi}}$, the fourth is the infinite norm of the Hamiltonian constraint, the fifth is the angular momentum of the spacetime, $J_{A D M}$, the sixth is the mass of the spacetime, $M_{A D M}$, and the last column is the apparent horizon mass, $M_{A H / M}$. The black holes have equal mass.

\begin{tabular}{ccccccc}
$d$ & $\Omega M_{\text {irr }}$ & $M_{\text {irr }}$ & Ham-Linf & $J_{A D M}$ & $M_{A D M} / M$ & $M_{A H} / M$ \\
\hline 6 & 0.168 & 2.63 & $3.33 \mathrm{e}-06$ & $1.75 \mathrm{e}-07$ & 2.59 & 1.31 \\
7 & 0.135 & 2.48 & $2.08 \mathrm{e}-06$ & $1.24 \mathrm{e}-07$ & 2.44 & 1.24 \\
8 & 0.112 & 2.39 & $1.40 \mathrm{e}-06$ & $9.83 \mathrm{e}-08$ & 2.35 & 1.20 \\
9 & 0.0949 & 2.33 & $9.97 \mathrm{e}-07$ & $8.16 \mathrm{e}-08$ & 2.29 & 1.17 \\
10 & 0.0817 & 2.29 & $7.38 \mathrm{e}-07$ & $6.98 \mathrm{e}-08$ & 2.25 & 1.14 \\
14 & 0.0505 & 2.19 & $1.33 \mathrm{e}-08$ & $1.83 \mathrm{e}-09$ & 2.17 & 1.09
\end{tabular}

The background is frozen in time for each solution to the Klein-Gordon equation. We need to specify the initial data for $\psi$ to represent an incoming wave of gravitational radiation:

$$
\psi=\psi_{0} e^{-\left(r-r_{0}\right)^{2} / \sigma^{2}} Y_{l=1, m=0}
$$

where $r_{0}$ is set to be $12 M$ away from the center of the black holes and $\sigma=2$.

\section{PROCEDURE}

This is a model problem intended to get at a feature of the BBH merger, the early formation of the $\mathrm{BH}$ potential. As the scalar field is scattered off of the $\mathrm{BBH}$ background, what do we expect it to tell us about the spacetime? There are two general cases to consider. When the black holes are far apart, we expect each individual black hole to act as an isolated black hole; exhibiting quasi-normal ringing. Thus, at large separations for equal-mass black holes, there are two characteristic frequencies, the frequency associated with the individual black holes' dominant QNM and the orbital frequency of the binary system itself. The second case is when the black holes have coalescenced and formed a single, more massive black hole. In this case there is a single frequency, that 
TABLE 2. Parameters for each run, where $\omega_{\text {qnm }}$ is the quasi-normal mode frequency found for each run.

\begin{tabular}{ccccc}
$d$ & $\omega_{\text {qnm }}$ & $\Omega M_{\text {irr }}$ & $M_{\text {irr }}$ & $M_{A D M} / M$ \\
\hline 6 & 0.130 & 0.168 & 2.63 & 2.59 \\
7 & 0.150 & 0.135 & 2.48 & 2.44 \\
8 & 0.202 & 0.112 & 2.39 & 2.35 \\
9 & 0.212 & 0.0949 & 2.33 & 2.29 \\
10 & 0.265 & 0.0817 & 2.29 & 2.25 \\
14 & 0.268 & 0.0505 & 2.19 & 2.17
\end{tabular}

of the QNM associated with the black hole. We use the ringing frequencies to predict how long we maintain individual, isolated black holes and then how quickly we see a single black hole form. However, the approximation we are making in using a sequence of initial data breaks down the closer the black holes get in the sequence.

The procedure to find the frequency of the dominant QNM is to fit the scattered pulse, $\psi$ to the expected functional form of quasi-normal ringdown, namely

$$
\psi \approx e^{-\tau+i \omega t}
$$

where $\omega$ is the real part of the frequency and $\tau$ is imaginary part associated with the decay time of the mode. We make the assumption that the fitted frequency is that of the dominant QNM, in this case the $l=1, m=0$ mode which has $\omega=0.293$. We then compute the mass of the black hole based on this fitted frequency such that

$$
M_{b h}=\frac{0.293}{w_{q n m}}
$$

Table (2) lists some of the same parameters as in Table (1) but now with the QNMfrequency, $\omega_{q n m}$ also given.

\section{RESULTS AND CONCLUSIONS}

Fig. (1) plots a series of waveforms from a sample of the different initial data sets parameterized by the separation, $d$. For reference, ISCO in this sequence of initial data is estimated to be located at $d=8 M$. The separations of the black holes are $d=14$ in the uppermost plot, $d=8$ in the middle and $d=6$ at the bottom. These separations represent well outside of the ISCO of this sequence of initial data at $d=14$, near ISCO at $d=8$ and within the predicted ISCO at $d=6$.

Fig. (2) is a plot of the fitted masses versus the orbital frequency of each initial data set. This fit is done assuming the frequency of the black hole is $\omega_{l=1, m=0}=0.293 / M_{b h}$ which is the frequency for a scalar perturbation. The plotted masses indicate that $90 \%$ of the mass of the spacetime is acting like a single black hole by the end of the experiment. More interestingly, before the predicted ISCO has been reached, the black holes loose their individual quasi-normal ringing which would indicate that some non-linear process was present, perhaps even an early potential formation. 


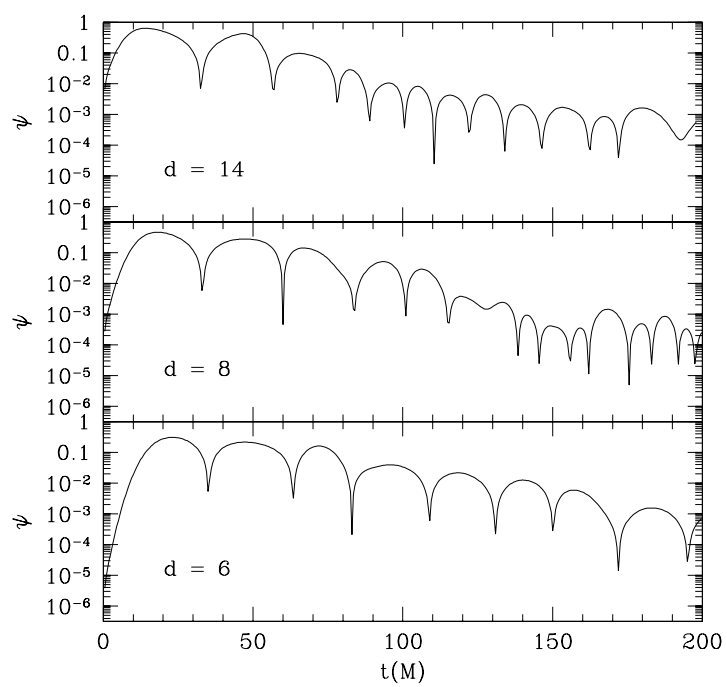

FIGURE 1. This plot is of $\log \psi$ versus time for three separations from Table (1). The uppermost plot is of the largest separation, the middle is at approximately ISCO and the bottommost is the closest binary separation, $d=6 M$.

As with photons and test particles, one can gain physical insight of numerically generated space times by studying the dynamics of scalar fields. Our numerical experiment supports the view that QNM ringing potential forms early on by the pre-ISCO loss of a fundamental frequency associated with the two, individual black holes. Secondly, this experiment also shows that an early, single black hole potential may form prior to an enveloping apparent horizon. This is seen by the $90 \%$ of the total mass already "ringing" before the single apparent horizon forms. No apparent horizon was found enveloping both black holes in any of the initial data sets.

The main limitation of the work is the application of the frozen background approximation. This approximation may be valid at the pre-ISCO part of the simulations, and we did not find a common apparent horizon in the initial data even at the closest separation. We will not know how accurate the approximation is until we use a scalar field to map the spacetime during a real evolution of the binary black holes. To do this correctly, we would use the time-evolution of the $\mathrm{BBH}$ problem such as the head-on, plunge or orbit collisions as the background taking one time step for each solution of the Klein-Gordon equation. In future work, we will return to this issue. We can use the type of experiment described in this proceeding to compare initial data sets and to probe how dependent the early formation of a potential might be on the choice of initial data, most importantly the freely specifiable parts and assumptions such as conformal flatness. Finally, this ex- 


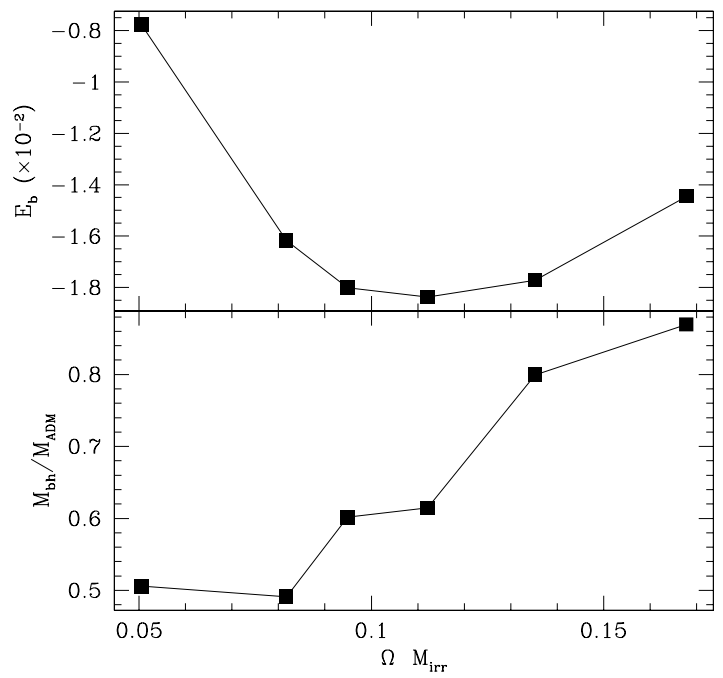

FIGURE 2. In this plot, the uppermost plot is the binding Energy, $E_{b}$ scaled by $10^{3}$ versus the orbital frequency, $\Omega M$ )irr for the quasi-circular sequence of initial data. The bottom plot is our results showing the fitted black hole mass $M_{b h} / M_{A D M}$ versus the orbital frequency. The black holes are furthest apart to the left $(d=14)$ and closest to the right $(d=6)$.

periment may give us insight into how early during the coalescence non-linear effects may become important.

\section{ACKNOWLEDGMENTS}

This work was supported by NSF grants PHY-9800973 and PHY-0114375. and PHY0354821. Work supported in part by the Center for Gravitational Wave Physics funded by the National Science Foundation under Cooperative Agreement PHY-0114375.

\section{REFERENCES}

1. L. Blanchet, Living Rev. Rel. 5, 2 (2002).

2. B. Brügmann, W. Tichy, and N. Jansen, Phys. Rev. Lett. 92, 211101 (2004), gr-qc/ 0312112.

3. M. Alcubierre, B. Brügmann, P. Diener, F. S. Guzmán, I. Hawke, S. Hawley, F. Herrmann, M. Koppitz, D. Pollney, E. Seidel, and J. Thornburg, Phys. Rev. D 72, 044004 (2005), URL http: //link.aps.org/abstract/PRD/v72/e044004,gr-qc/0411149.

4. F. Pretorius, Phys. Rev. Lett. 95, 121101 (2005), gr-qc/0507014. 
5. J. G. Baker, J. Centrella, D.-I. Choi, M. Koppitz, and J. van Meter, Gravitational wave extraction from an inspiraling configuration of merging black holes (2005), gr-qc / 0511103.

6. M. Campanelli, C. O. Lousto, P. Marronetti, and Y. Zlochower, Accurate evolutions of orbiting blackhole binaries without excision (2005), gr-qC/ 0511048.

7. P. Diener, F. Herrmann, D. Pollney, E. Schnetter, E. Seidel, R. Takahashi, J. Thornburg, and J. Ventrella, Accurate evolution of orbiting binary black holes (2005), gr-qc/ 0512108.

8. F. Herrmann, D. Shoemaker, and P. Laguna (2006), gr-qC/ 0601026.

9. M. Campanelli, C. O. Lousto, and Y. Zlochower, The last orbit of binary black holes (2006), gr-qc/0601091.

10. R. Price, and J. Pullin, Phys. Rev. Lett. 72, 3297 (1994).

11. G. Khanna, R. Gleiser, P. Laguna, O. Nicasio, H.-P. H.-P. Nollert, R. Price, and J. Pullin, Phys. Rev. Lett. 83, 3585 (1999).

12. J. Baker, M. Campanelli, C. Lousto, and R. Takahashi, Phys. Rev. D 65, 124012 (2002).

13. R. J. Gleiser, C. O. Nicasio, R. H. Price, and J. Pullin, Physical Review Letters 77, 4483-4486 (1996).

14. K. Kokkotas, and B. Schmidt, Living Rev. Rel. 2, 2 (1999).

15. L. Kidder, M. Scheel, S. Teukolsky, E. Carlson, and G. Cook, Phys. Rev. D 62, 084032 (2000).

16. M. A. Scheel, A. L. Erickcek, L. M. Burko, L. E. Kidder, H. P. Pfeiffer, and S. A. Teukolsky, Phys. Rev. D 69, 104006 (2004).

17. E. Gourgoulhon, P. Grandclément, and S. Bonazzola 65, 044020 (2002).

18. P. Grandclément, E. Gourgoulhon, and S. Bonazzola, Phys. Rev. D 65, 044021 (2002).

19. H. Pfeiffer, G. Cook, and S. Teukolsky, Phys. Rev. D 66, 024047 (2002).

20. H. Pfeiffer, L. Kidder, M. Scheel, and S. Teukolsky, Comput. Phys. Commun. 52, 253-274 (2003). 